\title{
A DIFFERENT LOOK AT THE RICHARDSON EXTRAPOLATION LEADING TO A NEW PROPOSITION
}

\author{
A. Soroushian \\ Structural Engineering Research Centre, International Institute of Earthquake Engineering and Seis- \\ mology (IIEES) \\ S. Lavasani (Farmaniyeh), North Dibajee, West Arghavan, No. 21, Tehran 19537, Iran
}

Keywords: Approximate Computation, Richardson Extrapolation, Convergence, Accuracy, Order of Accuracy, Taylor Series.

\begin{abstract}
Richardson extrapolation is proposed about a century ago and broadly accepted in the scientific community as a mean to accelerate the convergence of approximate computations. Many implementations, generalizations, and applications, of Richardson extrapolation, are reported in the literature. In this paper, considering the Taylor series expansion of approximate computations with respect to the algorithmic parameters, Richardson extrapolation is introduced as a tool towards higher accuracies, and the higher orders of accuracy are achieved as by-products. On this basis, a new proposition on Richardson extrapolation is stated, explained, and demonstrated, in an example.
\end{abstract}




\section{INTRODUCTION}

Richardson extrapolation [1,2], first introduced in the starting decades of the twentieth century, is a technique to accelerate sequential computations approach towards exact solutions, i.e. increase the order of accuracy and rate of convergence $[3,4]$. This is a significant achievement addressed in the literature as the most usefulness of Richardson extrapolation for practical computations or turning lead to gold $[5,6]$. Some of the applications of Richardson extrapolation are different advanced integration methods, e.g. the Romberg method, and a recent error estimation approach $[4,6]$. Order of accuracy and its increase would lose its meaning without convergence. However, convergence is an essentiality in approximate computations $[4,7,8]$ (and this highlights the applicability and versatility of Richardson extrapolation. Convergence implies the potential of arbitrary closeness to the exact solutions, i.e.

$$
\lim _{\lambda \rightarrow 0} \mathbf{U}^{a}=\mathbf{U}
$$

where, $\mathbf{U}^{a}$, and $\mathbf{U}$ respectively stand for the approximately computed and exact solutions, and $\lambda$ is the algorithmic parameter $[4,9,10]$. Pictorial representation of Eq. (1) is as noted in the convergence plot displayed in Fig. 1, where, $E$ stands for the error [11], i.e.

$$
E=\left\|\mathbf{U}^{a}-\mathbf{U}\right\|
$$

$q$ denotes the rate of convergence, such that

$$
q \leq q_{0}
$$

$q_{0}$ represents the order of accuracy and the difference between $q_{0}$ and $q$ is well studied and under control; e.g. see $[10,12,13]$, the mid-section of the convergence plot sloped $\cong q$ in Fig. 1 is the region of proper convergence [14], with a length that depending on the problem, computational method, and round-off, might even reduce to zero and vanish. The effect of Richardson extrapolation on convergence is as typically displayed in Fig. 2; see also [4]. As apparent in Fig. 2, Richardson extrapolation seems to have the potential to add the accuracy besides the order of accuracy. Based on this consideration, the attempt in this paper is to rederive the Richardson extrapolation as a technique to provide more accuracy, and to display the resulting higher order of accuracy, as a by-product, and finally, with attention to the essentialities of a specific application, to arrive at a new proposition on Richardson extrapolation, present an example, and discuss the practical consequences.

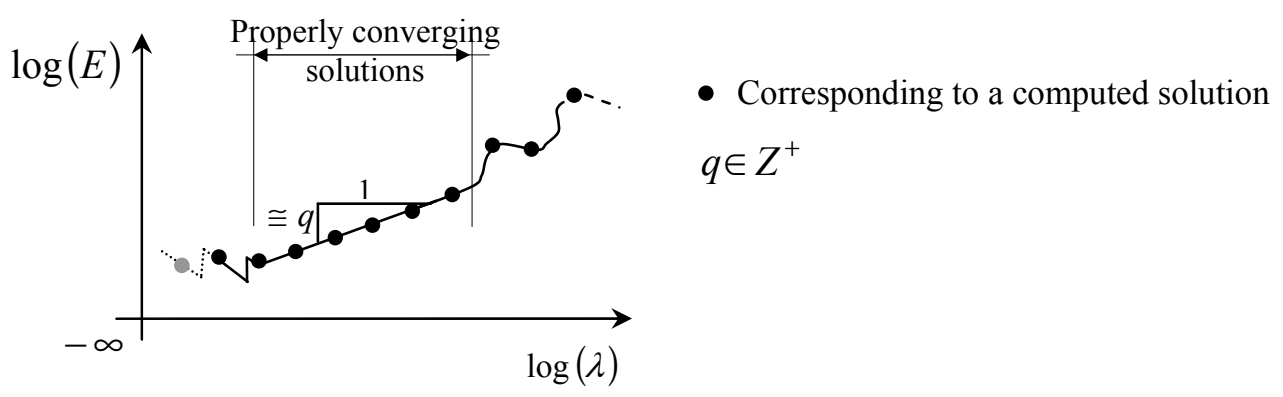

Figure 1: Typical changes of solutions errors with respect to the algorithmic parameters. 


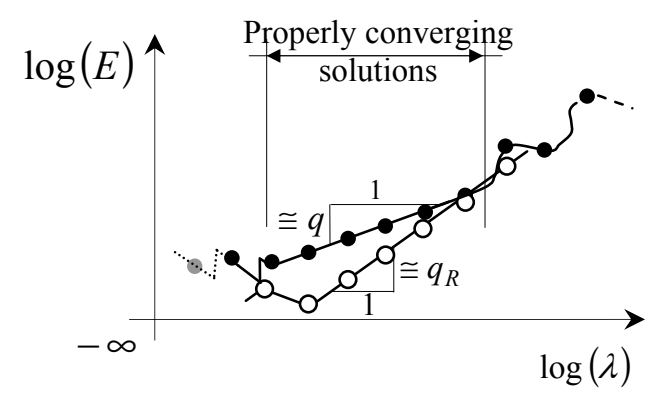

- Corresponding to a computed solution

○ Corresponding to a solution obtained from Richardson extrapolation

$q, q_{R} \in Z^{+} \quad, \quad q<q_{R}$

Figure 2: Typical effect of Richardson extrapolation on solutions convergence.

\section{AN ACCURACY BASIS FOR RICHARDSON EXTRAPOLATION}

Almost any approximate computation can be stated as noted below:

$$
\mathbf{U}^{a}=\mathbf{F}\left(\lambda, p_{1}, p_{2}, \ldots p_{n}, \gamma_{i=1,2, \ldots n^{\prime}}\right)
$$

where, $p_{i=1,2 \ldots . .}$ introduce the actual parameters defining the mathematical model, $\gamma_{i=1,2, \ldots n^{\prime}}$ define the details of the numerical method (model) and $\lambda$ stands for the algorithmic parameter, defined, via Eq. (1). By extending the definition of $\lambda$ to a continuously changing parameter and considering the fact that converging approximate computations are generally well behaved at close neighborhood of the exact solutions, we can express $\mathbf{U}^{a}$ in terms of the Taylor series expansion at $\mathbf{U}^{a}\left(\lambda \rightarrow 0^{+}\right)$, to arrive at

$$
\begin{aligned}
& \mathbf{U}^{a}=\mathbf{U}+\sum_{n=1}^{\infty} \frac{s_{n}}{n !} \lambda^{n} \\
& \mathbf{s}_{n}=\left.\frac{\partial^{n} \mathbf{F}}{\partial \lambda^{n}}\right|_{\lambda \rightarrow 0^{+}}
\end{aligned}
$$

$$
n=1,2,3, \ldots
$$

also equivalent to:

$$
\begin{aligned}
& \mathbf{U}^{a}-\mathbf{U}=\mathbf{C}_{0} \lambda^{q}+\mathbf{C}_{1} \lambda^{q+1}+\ldots \\
& \mathbf{C}_{i}=\frac{\mathbf{s}_{q+i}}{(q+i) !} \quad i=0,1,2,3, \ldots
\end{aligned}
$$

where,

$$
\begin{aligned}
& \mathbf{s}_{n}=\overline{\mathbf{O}} \\
& \mathbf{s}_{q} \neq \overline{\mathbf{O}}
\end{aligned} \quad, \quad n=1,2,3, \ldots q-1
$$

and even in a more detailed expression

$$
\begin{aligned}
& \mathbf{U}^{a}-\mathbf{U}=\mathbf{C}_{0}^{\prime} \lambda^{q_{0}}+\mathbf{C}_{1}^{\prime} \lambda^{q_{1}}+\mathbf{C}_{2}^{\prime} \lambda^{q_{2}}+\ldots \\
& \mathbf{C}_{i}^{\prime}=\frac{\mathbf{s}_{q_{i}}}{q_{i} !} \quad, \quad i=1,2, \ldots
\end{aligned}
$$

where, 


$$
\begin{aligned}
& \mathbf{s}_{n}=\overline{\mathbf{O}} \quad, \quad n \neq q_{i} \\
& \mathbf{s}_{n}=\left.\frac{\partial^{q_{i}} \mathbf{F}}{\partial \lambda^{q_{i}}}\right|_{\lambda \rightarrow 0^{+}} \quad, \quad n=q_{i} \quad i=1,2, \ldots
\end{aligned}
$$

and

$$
q_{0}<q_{1}<q_{2}<\ldots
$$

In view of Eqs. (6) or (8), for sufficiently small values of $\lambda$, the error as introduced in Eq. (2), mainly depend on $\mathbf{C}_{0}^{\prime}$, not on $\mathbf{C}_{1}^{\prime}, \mathbf{C}_{2}^{\prime}, \ldots$ In more detail, by gradually decreasing the value assigned to $\lambda$, the role of $\mathbf{C}_{i}^{\prime}$ decreases starting from the higher values of $i$ (with higher exponents of $\lambda$ ), and in some stage, it is only $\mathbf{C}_{0}^{\prime}$ that contributes $E$ (in continuation, the role of $\mathbf{C}_{0}^{\prime}$ will also vanish at convergence; see. Eq. (1)). This is in complete consistence with Fig. 1 and specifically the mid-section implying proper convergence. The role of $\mathbf{C}_{i=1,2, \ldots}^{\prime}$ is never zero; however, in view of Eq. (10), is negligible, depending on the problem at hand and the difference between $q_{0}$ and $q_{i=1,2, \ldots}$. Accordingly, if in defining a new computational method we can eliminate the term $\mathbf{C}_{0}^{\prime} \lambda^{q_{0}}$ from the right hand side of Eq. (8), and at the same time, guarantee a reasonably moderate change in $\mathbf{C}_{1}^{\prime}$, we would be able to expect more accuracy from the resultant of the elimination.

With this idea, consider two computations for one problem by one computational method,

$$
\begin{aligned}
& \mathbf{U}_{1}^{a}=\mathbf{U}^{a}\left(\lambda_{1}\right)=\mathbf{U}+\mathbf{C}_{0}^{\prime} \lambda_{1}{ }^{q_{0}}+\mathbf{C}_{1}^{\prime} \lambda_{1}{ }^{q_{1}}+\ldots \\
& \mathbf{U}_{2}^{a}=\mathbf{U}^{a}\left(\lambda_{2}\right)=\mathbf{U}+\mathbf{C}_{0}^{\prime} \lambda_{2}{ }^{q_{0}}+\mathbf{C}_{1}^{\prime} \lambda_{2}{ }^{q_{1}}+\ldots
\end{aligned}
$$

and define a new computation, ${ }^{R} \mathbf{U}_{1}^{a}$, for that problem, as stated below:

$$
{ }^{R} \mathbf{U}_{1}^{a}=\alpha \mathbf{U}_{1}^{a}+\beta \mathbf{U}_{2}^{a}
$$

where, $\alpha$ and $\beta$ are constants to be determined. In view of Eqs. (5) and (11), in order to preserve convergence to the exact solution $\mathbf{U}$, it is essential to be restricted to

$$
\alpha+\beta=1
$$

and since, in Eq. (8), $\mathbf{C}_{0}^{\prime} \neq \overline{\mathbf{O}}$ (see Eq. (1)), in order to omit the terms with exponent $q_{0}$, it is essential to satisfy

$$
\alpha \lambda_{1}^{q_{0}}+\beta \lambda_{2}{ }^{q_{0}}=0
$$

Consequently, from Eqs. (13) and (14),

$$
\alpha=\frac{\lambda_{2}{ }^{q_{0}}}{\lambda_{2}{ }^{q_{0}}-\lambda_{1}{ }^{q_{0}}} \quad, \quad \beta=\frac{\lambda_{1}{ }^{q_{0}}}{\lambda_{1}^{q_{0}}-\lambda_{2}{ }^{q_{0}}}
$$

and hence from Eq. (12),

$$
{ }^{R} \mathbf{U}_{1}^{a}=\frac{\lambda_{2}{ }^{q_{0}} \mathbf{U}_{1}^{a}-\lambda_{1}{ }^{q_{0}} \mathbf{U}_{2}^{a}}{\lambda_{2}{ }^{q_{0}}-\lambda_{1}{ }^{q_{0}}}=\frac{\lambda_{1}^{q_{0}} \mathbf{U}_{2}^{a}-\lambda_{2}{ }^{q_{0}} \mathbf{U}_{1}^{a}}{\lambda_{1}{ }^{q_{0}}-\lambda_{2}{ }^{q_{0}}}
$$


is a candidate for more accurate solution. The resulting change in the term $\mathbf{C}_{1}^{\prime} \lambda^{q_{1}}$ is as noted below ( ${ }^{R} \mathbf{C}_{1}^{\prime}$ is the coefficient of second converging term in the Taylor series expansion of ${ }^{R} \mathbf{U}_{1}^{a}$ with respect to $\lambda$; see also Eqs. (6), (8) or (11)) :

$$
\frac{\left\|{ }^{R} \mathbf{C}_{1}^{\prime}\right\|}{\left\|\mathbf{C}_{1}^{\prime}\right\|}=\frac{\left|\lambda_{1}^{q_{0}} \lambda_{2}^{q_{1}}-\lambda_{2}{ }^{q_{0}} \lambda_{1}{ }^{q_{1}}\right|}{\left|\lambda_{1}^{q_{0}}-\lambda_{2}{ }^{q_{0}}\right|} \frac{1}{\left|\lambda_{1}^{q_{1}}\right|}
$$

which considering

$$
\lambda_{1}=r \lambda_{2} \quad, \quad r>1
$$

leads to

$$
\frac{\left|{ }^{R} \mathbf{C}_{1}^{\prime}\right|}{\left|\mathbf{C}_{1}^{\prime}\right|}=\frac{\left|r^{q_{0}}-r^{q_{1}}\right|}{\left|r^{q_{0}}-1\right|} \frac{1}{\left|r^{q_{1}}\right|}<1
$$

that taking into account that $\lambda$ is small (at convergence) and $r$ and $q_{0}$ are finite, implies the decrease of $\mathbf{C}_{1}^{\prime}$, specifically in the mid-section in Fig. 1. Accordingly, for sufficiently small values of $\lambda$, specifically in the mid-section in Fig. 1, the ${ }^{R} \mathbf{U}_{1}^{a}$ defined in Eq. (16) will be more accurate than $\mathbf{U}_{1}^{a}$. For ${ }^{R} \mathbf{U}_{1}^{a}$ the Taylor series expansion can be stated as noted below:

$$
{ }^{R} \mathbf{U}_{1}^{a}=\mathbf{U}+\mathbf{C}_{1}^{\prime \prime} \lambda^{q_{1}}+\mathbf{C}_{2}^{\prime \prime} \lambda^{q_{2}}+\mathbf{C}_{3}^{\prime \prime} \lambda^{q_{3}}+\ldots
$$

where, $\mathbf{C}^{\prime \prime}$ can be simply computed in a process such that implemented in arriving at Eq. (19), and it is worth noting that, in consistence with Eqs. (17)-(19),

$$
\mathbf{C}_{i}^{\prime \prime}={ }^{R} \mathbf{C}_{i}^{\prime}
$$

In view of Eq. (10), Eq. (20) displays the higher order of accuracy for the computation:

$$
{ }^{R} \mathbf{U}_{1}^{a}={ }^{R} \mathbf{F}\left(\lambda, p_{i}, \ldots\right)
$$

Therefore, it is correct to claim that, ${ }^{R} \mathbf{U}_{1}^{a}$, being defined based on increasing accuracy (compared to $\mathbf{U}_{1}^{a}$ ), also leads to higher order of accuracy. With attention to Eq. (16), Eq. (21) coincides the Richardson extrapolation addressed in the literature [1-4], herein derived using the Taylor series expansion of a computation (in terms of the algorithmic parameter) on the basis of accuracy. A more rigorous explanation, especially for higher orders of Richardson extrapolation is the fact that as implied in Eqs. (12)-(16), for Richardson extrapolation of order $J, J$ terms are to be omitted from the Taylor series by solving a linear $(J+1) \times(J+1)$ algebraic system. However, with attention to the role and restrictions on $\lambda$ and $\lambda^{q_{i}}$, there is only one set of $\alpha_{1}, \alpha_{2}, \ldots \alpha_{J+1}$ to appropriately combine the solutions $\mathbf{U}^{a}\left(\lambda_{1}\right), \mathbf{U}^{a}\left(\lambda_{2}\right), \ldots \mathbf{U}^{a}\left(\lambda_{J+1}\right)$, and hence, any other appropriate combination of $\mathbf{U}^{a}\left(\lambda_{1}\right), \mathbf{U}^{a}\left(\lambda_{2}\right), \ldots \mathbf{U}^{a}\left(\lambda_{J+1}\right)$ should not exist, and consequently, the proposed approach and Richardson extrapolation lead to identical formulation regardless of the order of Richardson extrapolation.

Finally, it is worth noting that the presented derivation has also a geometrical explanation. In view of Fig. 1, for enhancement of accuracy in the proper convergence region of the convergence plot (more valid for smaller values of $\lambda$ ), there is no way but to make the slope of 
convergence trend more steep; the opposite is also correct. Albeit Richardson extrapolation is merely one alternative to enhance the accuracy and order of convergence; see also [15].

\section{THE RESULTING PROPOSITION}

In Section 2, Richardson extrapolation is introduced as the process of elimination of nonzero converging terms from the Taylor series expansion of the computation with respect to the algorithmic parameter, by linear combination of the computations at different values of the algorithmic parameters, while the elimination is started from the smallest exponents. This is summarized in the relations below:

$$
\begin{array}{r}
\mathbf{U}^{a}=\mathbf{F}\left(\lambda, \mathbf{C}_{0}\left(p_{1}, p_{2}, \ldots, \gamma_{i=1,2, \ldots n^{\prime}}\right), \mathbf{C}_{1}\left(p_{1}, p_{2}, \ldots, \gamma_{i=1,2, \ldots n^{\prime}}\right), \ldots\right) \\
\Downarrow \quad \text { Richardson Extrapolation of order } J \\
{ }_{J}^{R_{J}} \mathbf{U}^{a}={ }^{R_{J}} \mathbf{F}\left(\lambda, \mathbf{C}_{J}^{\prime}\left(p_{1}, p_{2}, \ldots, \gamma_{i=1,2, \ldots n^{\prime}}\right), \mathbf{C}_{J+1}^{\prime}\left(p_{1}, p_{2}, \ldots, \gamma_{i=1,2, \ldots n^{\prime}}\right), \ldots\right)
\end{array}
$$

where, $J$ is a positive integer, denoting the order of Richardson extrapolation, and meanwhile, the original computation can be considered as the Richardson extrapolation of order zero, i.e.

$$
{ }^{R_{0}} \mathbf{U}^{a}=\mathbf{U}^{a}
$$

all in consistence with the formulation presented for Richardson extrapolation [1-4]. In other words, for arriving at Richardson extrapolation of order $J$, the ordinary computation need to be considered at $J+1$ values of $\lambda$ and then the computations should be combined linearly, such that to preserve convergence and eliminate the $J$ terms $\mathbf{C}_{i}^{\prime} \lambda^{q_{i}}, i=0,1,2, \ldots J-1$ from the combination. From this perspective, it seems obvious that the $J$ terms can be eliminated in many ways, e.g. all together with one computation, one by one in $J$ separate stages, etc., all resulting in identical results $[6,16]$. Consequently, the $J$ th order Richardson extrapolation is identical to the $1^{\text {st }}$ order Richardson extrapolation of the $J-1$ th order Richardson extrapolation.

The above idea, or to say better claim, is true, because: (1) linear combination of linearly combined computations is a linear combination of the starting computations, (2) linear combination of $J+1$ terms such that to preserve convergence and omit $\mathbf{C}_{0}^{\prime} \lambda^{q_{0}}, \mathbf{C}_{1}^{\prime} \lambda^{q_{1}}, \ldots \mathbf{C}_{J-1}^{\prime} \lambda^{q_{J-1}}$, is unique, and hence, there is no difference, whether to arrive at Richardson extrapolation by directly omitting $\mathbf{C}_{0}^{\prime} \lambda^{q_{0}}, \mathbf{C}_{1}^{\prime} \lambda^{q_{1}}, \ldots \mathbf{C}_{J-1}^{\prime} \lambda^{q_{J-1}}$ terms using an appropriate linear combination, or first computing a Richardson extrapolation of order $J-m$

$$
0<m<J
$$

and omit $J-m$ terms and then compute the Richardson extrapolation of order $J$, by computing the Richardson extrapolation of order $m$ of the Richardson extrapolation of order $J-m$, i.e.

$$
{ }^{R_{J}} \mathbf{U}^{a}={ }^{R_{m}}\left(R_{J-m} \mathbf{U}^{a}\right)
$$

A direct extension of Eq. (26) is as stated below:

$$
R_{m_{1}+m_{2}+m_{3}+\ldots m_{N}} \mathbf{U}^{a}={ }^{R_{m_{1}}}\left({ }^{R_{m_{2}}}\left({ }^{R_{m_{3}}}\left(\ddots\left({ }^{R_{m_{N}}} \mathbf{U}^{a}\right)\right)\right)\right)
$$

Equation (27) is essential in formation of a recent convergence purification method $[17,18]$. 


\section{NUMERICAL STUDY}

Direct time integration is a versatile approach in analysis of structural systems against earthquake-induced ground motions [19, 20], and average acceleration [21], central difference [22], Wilson- $\theta$ [23-26], HHT [27], and C-H [28] methods, are of the broadly accepted time integration methods [20, 29-33]. In general, the responses obtained from time integration are approximations, and hence, the concepts of convergence, Richardson extrapolation, and that stated in Sections 2 and 3, are to be applicable; see also [3, 4, 34, 35]. Considering these, the structural system, introduced in Fig. 3(a) and Table 1, is analyzed against the ground motion introduced in Fig. 3(b), once by the average acceleration method and then again by the $\mathrm{C}-\mathrm{H}$ $(\rho=0.8)$ method. Since, in time integration analyses, the integration step size implies the algorithmic parameter [10], i.e.

$$
\lambda=\Delta t
$$

the results of Richardson extrapolation of second order is first computed directly, in view of the relation below [4]:

$$
{ }^{R_{2}} \mathbf{U}^{a}=\frac{r^{q_{0}+q_{1}} \mathbf{U}_{3}^{a}-\left(r^{q_{0}}+r^{q_{1}}\right) \mathbf{U}_{2}^{a}+\mathbf{U}_{1}^{a}}{r^{q_{0}+q_{1}}-\left(r^{q_{0}}+r^{q_{1}}\right)+1}
$$

where,

$$
\begin{gathered}
\frac{\lambda_{1}}{\lambda_{2}}=\frac{\lambda_{2}}{\lambda_{3}}=r=\text { const. }=2>1 \\
\mathbf{U}_{3}^{a}=\mathbf{U}^{a}\left(\lambda=\lambda_{3}\right), \quad \mathbf{U}_{2}^{a}=\mathbf{U}^{a}\left(\lambda=\lambda_{2}\right), \quad \mathbf{U}_{1}^{a}=\mathbf{U}^{a}\left(\lambda=\lambda_{1}\right)
\end{gathered}
$$

and the values of $q_{0}$ and $q_{1}$ for the two integration methods are as noted below [36]:

$$
\begin{array}{llll}
\text { Average acceleration : } & q_{0}=2 \quad, \quad q_{1}=4 \\
\mathrm{CH}(\rho=0.8): & q_{0}=2 \quad, \quad q_{1}=3
\end{array}
$$

(a)

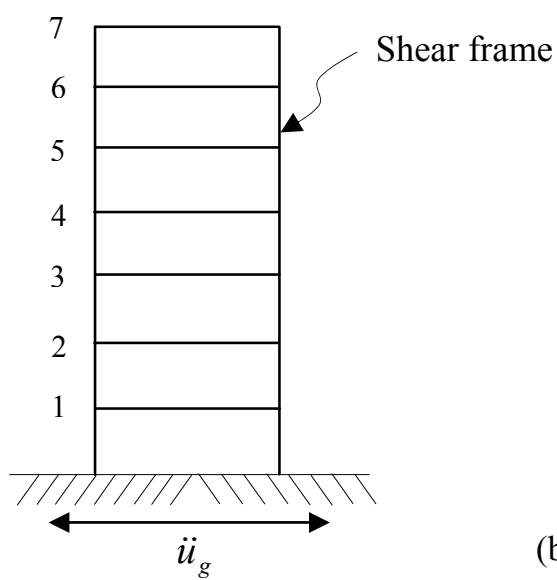

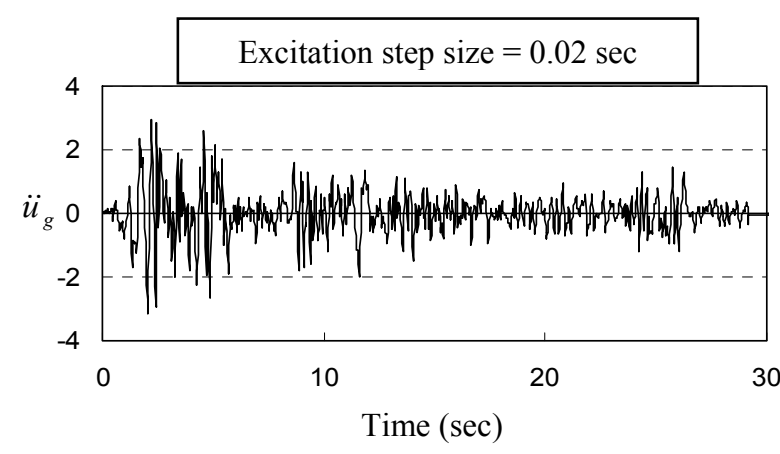

b)

Figure 3: The structural system in the numerical study: (a) the structure, (b) the ground motion.

\begin{tabular}{cccccccc}
\hline Floor & 1 & 2 & 3 & 4 & 5 & 6 & 7 \\
\hline Mass $\times 10^{-3}(\mathrm{Kg})$ & 2068 & 2064 & 2060 & 2056 & 2052 & 2048 & 2044 \\
\hline Stiffness $\times 10^{-6}(\mathrm{~N} / \mathrm{m})$ & 840 & 820 & 700 & 680 & 660 & 640 & 620 \\
\hline
\end{tabular}

Table 1: Main properties of the structure in Fig. 3(a). 
and then by implementing the first order Richardson extrapolation (see Eq. (16) and [4]) to the resultant of first order Richardson extrapolation. The results together with the convergence trends are reported in Tables 2 and 3 and Figs. 4 and 5, where, the response under consideration is as noted below

$$
\mathbf{U}^{a}=\left\{\begin{array}{c}
\text { Maximum Top Displacement } \\
\text { Final Base Shear }
\end{array}\right\}
$$

addressed as the top and bottom values in each cell of Tables 2 and 3. By comparing the last two columns in each table, it becomes apparent that Eq. (26) holds for both of the two time integration computations. This is a simple clear evidence for the validity of Eqs. (26) and (27). Similarly, and besides the theoretical discussion presented in Section 2, Figs. 4 and 5 imply the validity of the addressed accuracy basis and indeed the equivalence between the two apbases (accuracy-based and order of accuracy-based) leading to Richardson extrapolation.

\begin{tabular}{|c|c|c|c|c|c|c|}
\hline$\Delta t$ & $\mathbf{U}_{1}^{a}$ & $\mathbf{U}_{2}^{a}$ & $\mathbf{U}_{3}^{a}$ & $R_{1} \mathbf{U}^{a}$ & $R_{1}\left(R_{1} \mathbf{U}^{a}\right)$ & $R^{R_{2}} \mathbf{U}^{a}$ \\
\hline \multirow{2}{*}{0.02} & - & - & 0.26570572 & - & - & - \\
\hline & - & - & -6633587.8 & - & - & - \\
\hline \multirow{2}{*}{0.01} & - & 0.26570572 & 0.27267107 & 0.27499285 & - & - \\
\hline & - & -6633587.8 & -10209.648 & 2197583.07 & - & - \\
\hline \multirow{2}{*}{0.005} & 0.26570572 & 0.27267107 & 0.27284983 & 0.27290942 & 0.27277053 & 0.27277053 \\
\hline & -6633587.8 & -10209.648 & 848092.950 & 1134193.82 & 1063301.20 & 1063301.20 \\
\hline \multirow{2}{*}{0.0025} & 0.27267107 & 0.27284983 & 0.27298766 & 0.27303360 & 0.27304188 & 0.27304188 \\
\hline & -10209.648 & 848092.950 & 500907.284 & 385178.729 & 335244.390 & 335244.390 \\
\hline \multirow{2}{*}{0.00125} & 0.27284983 & 0.27298766 & 0.27303644 & 0.27305270 & 0.27305398 & 0.27305398 \\
\hline & 848092.950 & 500907.284 & -929445.92 & -1406230.3 & -1525657.6 & -1525657.6 \\
\hline \multirow{2}{*}{0.000625} & 0.27298766 & 0.27303644 & 0.27304956 & 0.27305393 & 0.27305401 & 0.27305401 \\
\hline & 500907.284 & -929445.92 & -1276705.6 & -1392458.8 & -1391540.7 & -1391540.7 \\
\hline \multirow{2}{*}{0.0003125} & 0.27303644 & 0.27304956 & 0.27305302 & 0.27305417 & 0.27305419 & 0.27305419 \\
\hline & -929445.92 & -1276705.6 & -1360811.4 & -1388846.7 & -1388605.9 & -1388605.9 \\
\hline
\end{tabular}

Table 2: Comparison of the two approaches in computation of second order Richardson extrapolation in analysis of the system in Fig. (3) by the average acceleration method.

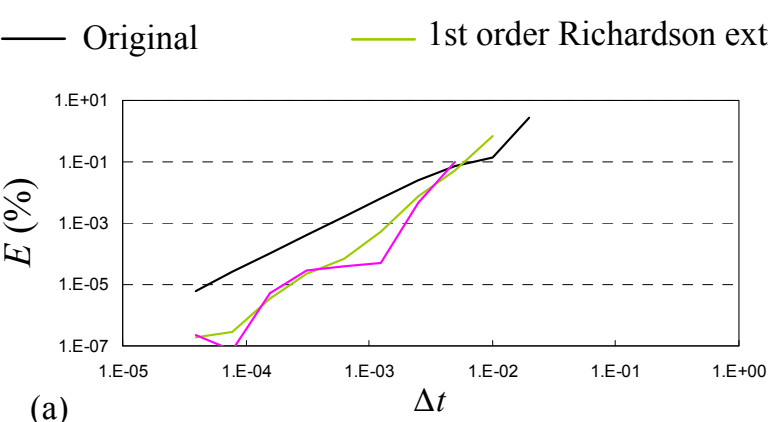

(a) $\Delta t$ (b)

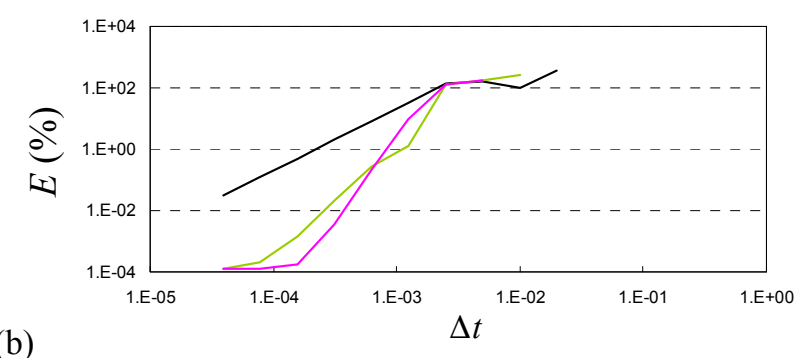

Figure 4: Convergence plots corresponding to Table 2: (a) Maximum top displacement, (b) Final base shear. 


\begin{tabular}{|c|c|c|c|c|c|c|}
\hline$\Delta t$ & $\mathbf{U}_{1}^{a}$ & $\mathbf{U}_{2}^{a}$ & $\mathbf{U}_{3}^{a}$ & ${ }^{R_{1}} \mathbf{U}^{a}$ & $R_{1}\left(R_{1} \mathbf{U}^{a}\right)$ & ${ }^{R_{2}} \mathbf{U}^{a}$ \\
\hline \multirow{2}{*}{0.02} & - & - & 0.26515318 & - & - & - \\
\hline & - & - & -6602365.4 & - & - & - \\
\hline \multirow{2}{*}{0.01} & - & 0.26515318 & 0.27265911 & 0.27516110 & - & - \\
\hline & - & -6602365.4 & -1307192.0 & -1307191.9 & - & - \\
\hline \multirow{2}{*}{0.005} & 0.26515318 & 0.27265911 & 0.27284168 & 0.27290253 & 0.27257988 & 0.27257988 \\
\hline & -6602365.4 & -1307192.0 & 413986.707 & 987712.942 & 1315556.51 & 1315556.51 \\
\hline \multirow{2}{*}{0.0025} & 0.27265911 & 0.27284168 & 0.27298421 & 0.27303173 & 0.27305018 & 0.27305018 \\
\hline & -1307192.0 & 413986.707 & 600791.175 & 663059.330 & 616680.243 & 616680.243 \\
\hline \multirow{2}{*}{0.00125} & 0.27284168 & 0.27298421 & 0.27303546 & 0.27305254 & 0.27305551 & 0.27305551 \\
\hline & 413986.707 & 600791.175 & -903083.30 & -1404374.8 & -1699722.5 & -1699722.5 \\
\hline \multirow{2}{*}{0.000625} & 0.27298421 & 0.27303546 & 0.27304931 & 0.27305393 & 0.27305412 & 0.27305412 \\
\hline & 600791.175 & -903083.30 & -1270409.8 & -1392852.0 & -1391205.9 & -1391205.9 \\
\hline \multirow{2}{*}{0.0003125} & 0.27303546 & 0.27304931 & 0.27305296 & 0.27305418 & 0.27305421 & 0.27305421 \\
\hline & -903083.30 & -1270409.8 & -1359262.9 & 423470.293 & 682944.905 & 682944.905 \\
\hline
\end{tabular}

Table 3: Comparison of the two approaches in computation of second order Richardson extrapolation in analysis of the system in Fig. (3) by the C-H $(\rho=0.8)$ method.

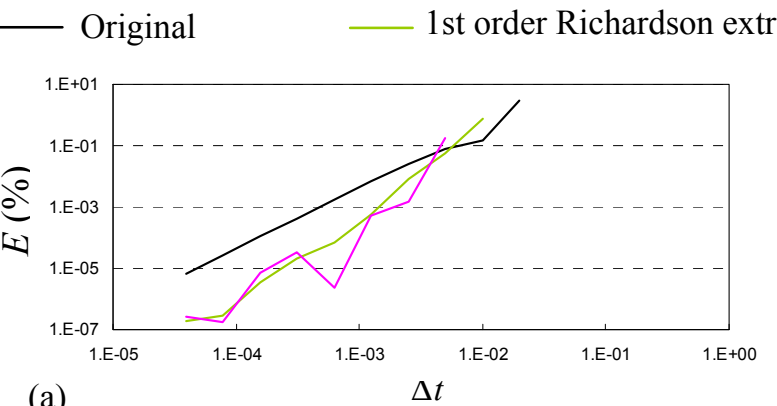

(a)

Figure 5: Convergence plots corresponding to Table 3 : (a)

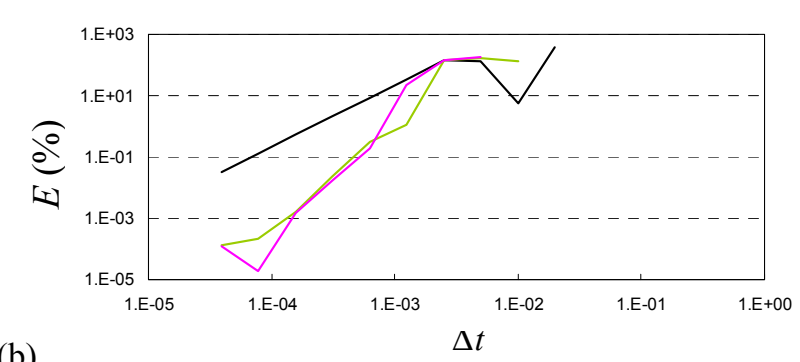

(b)

(a) Maximum top displacement, (b) Final base shear. 2nd order Richardson extrapolation

\section{CONCLUSION}

- Richardson extrapolation can be considered as the process of elimination of terms from the Taylor series expansion of the computation with respect to the algorithmic parameter, starting from the smallest exponents of the algorithmic parameter in the Taylor series. From this point of view, further accuracy in the proper convergence region as well as higher order of accuracy can be considered as the basis of Richardson extrapolation.

- Richardson extrapolation of order $J$, where $J$ is a positive integer larger than one, can be obtained either directly or by first computing the Richardson extrapolation of a positive integer less than $J$, i.e. $m$, and then computing the Richardson extrapolation of order $J-m$ of the resultant. This can be extended; the number of the stages of implementation of Richardson extrapolation can be as large as $J$, at which case, in view of Eq. (26), Richardson extrapolation will be implemented sequentially for $J$ times. 


\section{REFERENCES}

[1] L.F. Richardson, J.A. Gaunt, The deferred approach to the limit. Philosophical Transactions of the Royal Society of London, 226, 299-361, 1927.

[2] L.F. Richardson. The approximate arithmetical solution by finite differences of physical problems including differential equations, with an application to the stresses in a masonry dam. Philosophical Transactions of the Royal Society of London, 210, 307-357, 1910 .

[3] C. Brezenski, M. Redivo Zagalia, Extrapolation methods: theory and practice. NorthHolland, 1991.

[4] A. Soroushian, P. Wriggers, J. Farjoodi, Asymptotic upper bounds for the errors of Richardson extrapolation with practical application in approximate computations. International Journal for Numerical Methods in Engineering, 80, 565-595, 2009.

[5] G. Birkhoff, G.C. Rota, Ordinary differential equations. John Wiley \& Sons, 1978.

[6] W.H. Press, S.A. Teukolsky, W.T. Vetterling, B.P. Flannery, Numerical recipes in Fortran The Art of Scientific Computing, 4th Edition. Cambridge University Press, 1992.

[7] P. Henrici, Discrete variable methods in ordinary differential equations, Prentice-Hall, 1962.

[8] J.C. Strikwerda, Finite difference schemes and partial differential equations. Wadsworth \& Books/Cole, 1989.

[9] C.W. Gear, Numerical initial value problems in ordinary differential equations. Prentice-Hall, 1971.

[10] A. Soroushian, A technique for time integration with steps larger than the excitation steps. Communications in Numerical Methods in Engineering, 24, 2087-2111, 2008.

[11] A. Ralston, P. Rabinowitz, First course in numerical analysis. McGraw-Hill, 1978.

[12] W.L. Wood, Practical time stepping schemes. Clarendon, 1990.

[13] S.N. Penry, W.L. Wood, Comparison of some single-step methods for the numerical solution of the structural dynamic equation. International Journal for Numerical Methods in Engineering, 21, 1941-1955, 1985.

[14] A. Soroushian, Proper convergence a concept new in science and important in engineering. D. Tsahalis ed. $4^{\text {th }}$ International Conference from Scientific Computing to Computational Engineering (4 $\left.{ }^{\text {th }} I C-S C C E\right)$, Athens, Greece, July 7-10, 2010.

[15] A. Soroushian, Convergence with non-integer rates. D. Tsahalis ed. $4^{\text {th }}$ International Conference from Scientific Computing to Computational Engineering ( $4^{\text {th }}$ IC-SCCE), Athens, Greece, July 7-10, 2010.

[16] G.H. Golub, C.F. Van Loan, Matrix Computations, Third Edition. John Hopkins University Press, 1996.

[17] A. Soroushian, Purification of convergence an approach towards reliable error evaluation. E. Onate, X. Oliver, A. Huerta eds. $11^{\text {th }}$ World Congress on Computational Mechanics (WCCM XI), Barcelona, Spain, July 20-25, 2014.

[18] A. Soroushian, Purification of convergence: from definition to consequences. (to be submitted) 
[19] J. Chung, G.M. Hulbert, A family of single-step Houbolt time integration algorithms for structural dynamics. Computer Methods in Applied Mechanics and Engineering, 118, 111, 1994.

[20] A.K. Chopra, Dynamics of structures: theory and application to earthquake engineering. Prentice-Hall, 1995.

[21] M.N. Newmark, A method of computation for structural dynamics. Journal of Engineering Mechanics (ASCE), 85, 67-94, 1959.

[22] R.W. Clough, Numerical integration of equations of motion. Lectures on Finite Element Methods in Continuum Mechanics, University of Alabama, pp. 525-533, 1973.

[23] E.L. Wilson, A computer program for the dynamic stress analysis of underground structures, Report No. 68-1. University of California, Berkeley, 1968.

[24] K.J. Bathe, E.L. Wilson, Stability and accuracy analysis of direct integration methods. Earthquake Engineering and Structural Dynamics, 1, 283-291, 1973.

[25] E.L. Wilson, I. Farhoomand, K.J. Bathe, Non-linear dynamic analysis of complex structures. Earthquake Engineering and Structural Dynamics, 1, 241-252, 1973.

[26] A. Soroushian, J. Farjoodi, K. Bargi, M. Rajabi, A. Saaed, M. Arghavani, M.M. Sharifpour, Two versions of the Wilson- $\theta$ time integration method. J. Náprstek, J. Horáček, M. Okrouhlík, B. Marvalová, F. Verhulst, J.T. Sawicki eds. $10^{\text {th }}$ Interantioanl Conferecne on Vibration Problems. (ICoVP 2011), Prague, Czech Republic, September 5-8, 2011.

[27] H.M. Hilber, T.J.R. Hughes, R.L. Taylor, Improved numerical dissipation for time integration algorithms in structural dynamics. Earthquake Engineering and Structural Dynamics, 5, 283-292, 1977.

[28] J. Chung, G.M. Hulbert, A time integration algorithm for structural dynamics with improved numerical dissipation: the generalized- $\alpha$ method. Journal of Applied Mechanics (ASME), 60, 371-375, 1993.

[29] T.J.R. Hughes, The finite element method: linear static and dynamic finite element analysis. Prentice-Hall, 1987.

[30] K.J. Bathe, Finite element procedures. Prentice-Hall, 1996.

[31] R.W. Clough, J. Penzien, Dynamics of structures. McGraw-Hill, 1993.

[32] J.L. Humar, Dynamics of structures. Prentice-Hall, 1990.

[33] R.R. Craig, Structural dynamics: an introduction to computer methods. John Wiley \& Sons, 1981.

[34] M.N. Bismarck-Nasr, A.M. De Oliveira, On enhancement of accuracy in direct time integration dynamic response problems. Earthquake Engineering and Structural Dynamics, 20, 699-703, 1991.

[35] Belytschko and Hughes T. Belytschko, T.J.R. Hughes, Computational methods for transient analysis, Elsevier, 1983.

[36] A. Soroushian, Effect of Richardson extrapolation on the convergence of some time integration methods. D. Tsahalis ed. $6^{\text {th }}$ International Conference from Scientific Computing to Computational Engineering ( $\left.6^{\text {th }} I C-S C C E\right)$, Athens, Greece, July 9-12, 2014. 